\title{
Novos Compostos, Grandes Desafios!
}

\author{
Tânia S. Morais* \\ Centro de Ciências Moleculares e Materiais, Departamento de Química e Bioquímica \\ Faculdade de Ciências da Universidade de Lisboa \\ tsmorais@fc.ul.pt
}

\begin{abstract}
cancro é uma das principais causas de morte em todo o mundo e segundo a Organização Mundial de Saúde este número aumentará de 7,9 para 11,5 milhões até 2030. Embora existam muitos fármacos em uso clínico, nenhum é suficientemente eficiente, devido aos baixos índices terapêuticos e a um amplo espectro de efeitos secundários graves. O desenvolvimento de novos fármacos anticancerígenos baseados em novos modos de aç̧ão é de extrema urgência. Assim, o meu trabalho de doutoramento consistiu no desenvolvimento de novos complexos organometálicos de ruténio e no estudo do seu potencial como novos agentes antineoplásicos com novos modos de acção, pretendendo dar, deste modo, uma contribuição para a resolução deste grave problema.
\end{abstract}

Cancro, tumor maligno e neoplasia são termos genéricos usados para um largo número de doenças que afectam qualquer parte do organismo. O cancro é caracterizado por um crescimento descontrolado de células, seguido por invasão das células saudáveis e disseminação por outros órgãos (metástases).

O cancro é uma das principais causas de morte no mundo (segunda na Europa) e o número total de casos continua a aumentar. Segundo a Organização Mundial de Saúde o número de mortes deverá aumentar 45\% até 2030, passando de 7,9 milhões para 11,5 milhões [1]. Nos homens, os cancros da próstata, pulmão e colorrectal são os mais vulgares, sendo responsáveis por $50 \%$ das mortes por cancro [2]. Nas mulheres, os tipos de cancro predominantes são mama, pulmão e colorrectal (Figura 1).

As estratégias para controlar o cancro, após remoção cirúrgica, são focadas em tratamentos citotóxicos convencionais: radioterapia e quimioterapia. Ambos apresentam baixos índices terapêuticos e um amplo espectro de efeitos secundários graves [3]. Os efeitos secundários são intensificados uma vez que a maioria dos fármacos exibe pouca ou nenhuma selectividade para as células cancerígenas, não poupando assim as células saudáveis [4]. Outra limitação é o desenvolvimento de resistência ao fármaco, em parte devido à sua pobre penetração no tumor e ao desenvolvimento de metástases [4]. Portanto, é de extrema urgência a descoberta de novos fármacos anticancerígenos baseados em novos modos de acção com maior selectividade e eficiência contra as metástases.

\section{O RUTÉNIO NA TERAPIA DO CANCRO}

Na procura de novos metalofármacos como agentes anticancerígenos, a química do ruténio tem sido alvo de muita atenção devido aos resultados promissores obtidos para

* Vencedora do PYCA-Gradiva 2014

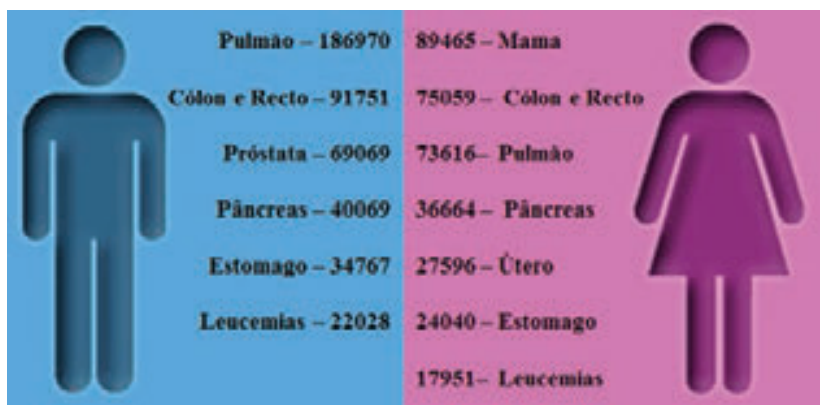

Figura 1 - Número de mortes previstas para a União Europeia para o ano de 2013 [2]

algumas famílias de complexos inorgânicos octaédricos de Ru(III). Neste contexto, os complexos de coordenação NAMI-A e KP1019 são os exemplos mais representativos, sendo os únicos complexos de ruténio que entraram em testes clínicos até à data; aparentemente o NAMI-A abandonou estes testes devido a problemas de estabilidade. Embora inicialmente os complexos de ruténio fossem concebidos com o objectivo de contornar os efeitos secundários das quimioterapias de platina, o sucesso de alguns destes compostos de ruténio como inibidores de metástases salientou o seu potencial no campo das novas terapias. Até ao presente, não foram ainda encontrados fármacos capazes de controlar o crescimento das metástases, sendo este problema uma das principais dificuldades na actual luta contra o cancro.

A estratégia de síntese dos rutenofármacos tem sido baseada nos modelos da platina levando a compostos que conduzam à morte celular por interacção com o ADN, embora visando preferencialmente novos mecanismos de acção, de modo a vencer a resistência das células tumorais. Neste contexto, vários complexos organometálicos de ruténio(II) foram sintetizados tendo em vista o ADN como alvo principal.

Assim, foram desenvolvidas e estudadas, fundamentalmente, duas famílias de complexos organometálicos ruténio(II)-areno (Figura 2) por Sadler et. al [5,6] (complexos RM) e Dyson et. al $[7,8]$ (complexos RAPTA), cuja estrutura 
básica assenta numa geometria em banco de piano e a sua reactividade baseia-se na saída dos ligandos cloreto, tal como acontece com a cisplatina. Embora estas duas famílias de compostos sejam estruturalmente semelhantes têm comportamentos muito diferentes. Enquanto os complexos RM apresentam uma boa actividade citotóxica in vitro, os RAPTA apresentam baixa citotoxicidade, mas mostram ser bastante eficientes contra as metástases. Meggers et. al [9] desenvolveram uma terceira família de compostos organometálicos (complexos DW) que envolve ligandos orgânicos piridocarbazolo (imitam a estaurosporina) coordenados ao fragmento organometálico Ru(II)-ciclopentadienilo (Figura 2), que se revelaram potentes inibidores das proteínas cinases GSK-3 e Pim-1, mostrando assim a sua potencialidade como agentes antitumorais. gasosa por espectrometria de massa (ESI-MS), realizados com vários complexos desta família de estrutura geral $\left[\mathrm{RuCp}(\mathrm{PP})_{\mathrm{n}} \mathrm{L}\right]\left[\mathrm{CF}_{3} \mathrm{SO}_{3}\right]$ (onde PP = fosfano, $\mathrm{L}=$ ligando heteroaromático e $n=1$ ou 2); verificou-se que a ligação Ru-Cp é extremamente estável, impossível de dissociar mesmo para valores muito altos de energia [10].

Esta estrutura geral em banco de piano permite-nos jogar com os vários grupos nas "pernas do piano"; neste caso foram escolhidos ligandos fosfanos (mono ou bidentados) e ligandos heretoaromáticos coordenados por átomos de $\mathrm{N}$, $\mathrm{O}, \mathrm{S}$, etc., onde a coordenação pode ser mono ou bidentada. O design destes compostos pode levar a uma significativa diversidade estrutural, uma vez que podemos intervir nas várias partes da molécula, nomeadamente na funcionaliza-
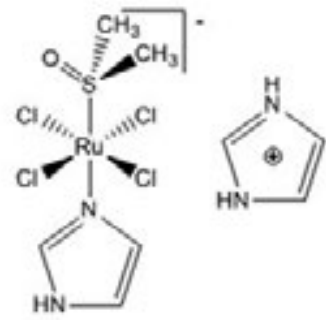

NAMI-A
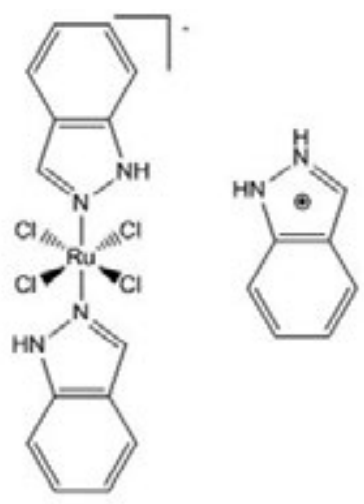

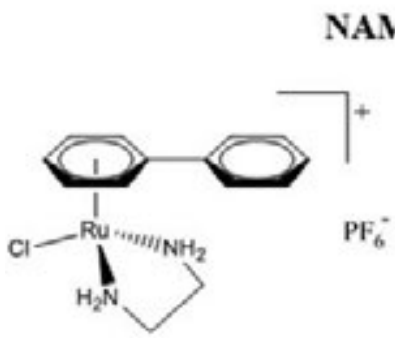

Complexos RM

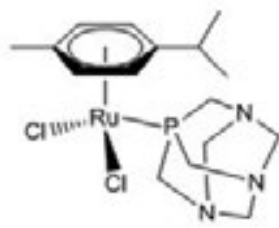

Complexos RAPTA
KP1019

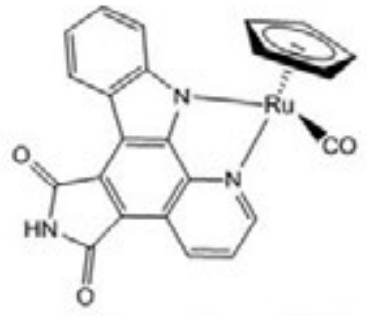

Complexos DW

Figura 2 - Estruturas químicas de alguns complexos de ruténio

\section{A NOSSA ESTRATÉGIA}

A nossa estratégia para o desenvolvimento de novos agentes antitumorais passa por desenhar compostos que interactuem com outros alvos para além do ADN, de forma a constituir novas alternativas de acção e ultrapassar as limitações dos fármacos existentes no mercado. Neste contexto, desenvolvemos uma família de compostos organometálicos de ruténio, para os quais se esperam diferentes mecanismos de acção devido à ausência dos grupos cloretos existentes nos complexos descritos na bibliografia atrás referidos.

A ligação ruténio-carbono é construída por um grupo ciclopentadienilo $\left(\eta^{5}-\mathrm{C}_{5} \mathrm{H}_{5}=\mathrm{Cp}\right)$, em que a carga negativa dá uma estabilidade electrostática adicional para o fragmento "RuCp", em comparação com o seu equivalente "Ru-areno" (Figura 3) em que se baseiam os compostos RM e RAPTA atrás referidos. A estabilidade do fragmento "RuCp" foi corroborada pelos nossos estudos em fase
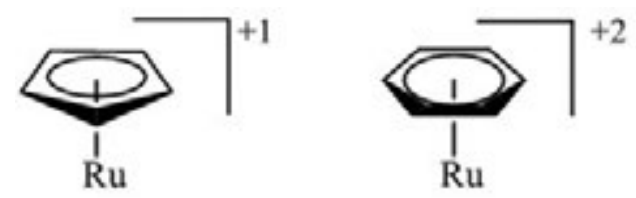

Figura 3 - Construção de estruturas em banco de piano com base em grupos ciclopentadienilo e areno

ção do anel Cp, na variação dos números de coordenação e natureza dos coligandos e do próprio contra-ião. Algumas destas características são extremamente importantes para controlar a solubilidade dos compostos e optimizar a actividade biológica.

Assim, usando esta estratégia foram sintetizados vinte e seis compostos de ruténio, com diferentes fosfanos, contra-iões e ligandos heteroaromáticos, e diferentes modos de coordenação de forma a controlar/optimizar algumas características como estabilidade, solubilidade em meio aquoso, citotoxicidade e selectividade (Figura 4). 

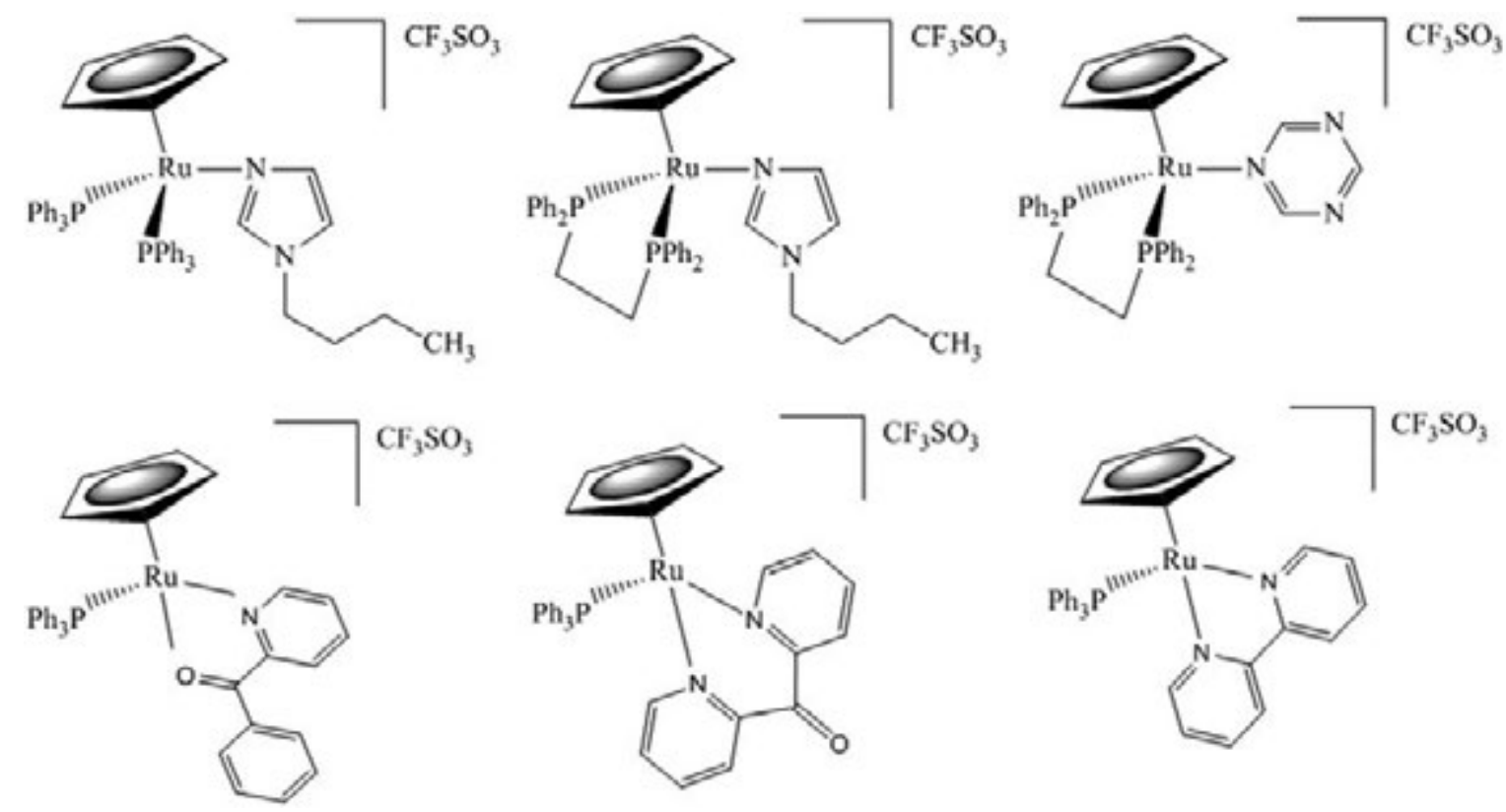

Figura 4 - Exemplo de várias estruturas de complexos sintetizados mostrando as diferentes formas de coordenação dos ligandos e a sua variedade estrutural

\section{AcTIVIDADE CITOTÓXICA}

A actividade citotóxica de todos os complexos foi avaliada in vitro em várias linhas celulares tumorais humanas, nomeadamente A2780 (carcinoma do ovário), A2780CisR (carcinoma do ovário resistente à cisplatina), MCF7 (adenocarcinoma da mama, dependente de estrogénio), MDAMB231 (adenocarcinoma da mama, independente de estrogénio), PC3 (carcinoma da próstata) e HT29 (carcinoma do cólon). Estas linhas foram escolhidas de modo a englobar diferentes sensibilidades ao tratamento com metalofármacos, incluindo os tipos de cancros mais diagnosticados nos homens (cancro da próstata) e nas mulheres (cancro da mama). Todos os compostos mostraram ser alta ou moderadamente citotóxicos in vitro para as seis linhas tumorais humanas estudadas, com valores de $\mathrm{IC}_{50}$ da ordem micro e nanomolar. Na sua maioria são bastante mais citotóxicos que a cisplatina (metalofármaco em uso clínico). Foram também realizados estudos na presença de uma linha celular saudável, sendo de notar que os compostos mostraram possuir baixa citotoxicidade para a linha celular saudável V79, com valores de $\mathrm{IC}_{50} 60$ a 130 vezes mais elevados que para as células cancerígenas, revelando assim uma importante selectividade intrínseca.

\section{TRANSPORTE NO SANGUE}

Numa primeira abordagem para compreender o perfil farmacocinético destes compostos, foi avaliada a sua interacção com proteínas do soro humano (albumina e transferrina) pois a ligação proteína-fármaco influencia a distribuição e as propriedades farmacocinéticas de um fármaco. Estes estudos revelaram a formação de aductos estáveis com estas proteínas de transporte, condição essencial para serem transportados até aos seus alvos.

Foram também realizados ensaios de viabilidade celular na presença destas duas proteínas de forma a avaliar se a sua ligação aos complexos podia influenciar a actividade citotóxica dos mesmos. Foi possível verificar que a formação destes aductos não afecta as propriedades citotóxicas dos complexos, pelo contrário, em alguns casos, a ligação à transferrina parece facilitar a entrada nas células.

\section{Alvos celulares}

A informação relativa à distribuição dos fármacos e à sua concentração nas células tumorais é da maior importância para a compreensão dos mecanismos de acção. Os fármacos, para além de entrarem nas células, devem concentrar-se nos compartimentos celulares onde estão localizados os alvos. Os nossos estudos realizados por Espectrometria de Massa com fonte de Plasma Indutivamente Acoplada (ICP-MS) permitiram identificar os compartimentos celulares em que o ruténio fica retido e também quantificar a sua percentagem em cada compartimento. Foi possível verificar que os nossos complexos de ruténio ficam retidos preferencialmente na membrana celular, embora uma pequena percentagem chegue mesmo ao citosol, núcleo e citoesqueleto.

Quando se avalia o potencial terapêutico de um complexo é essencial compreender o mecanismo de entrada nas células, o que condiciona a percentagem de complexo que é internalizado bem como a sua localização na célula. Para estudar os mecanismos de captação celular recorreu-se ao uso de moduladores de entrada nas células. Demonstrou-se que estes complexos entram nas células por um processo dependente de energia (mecanismo activo) identificado como endocitose, sendo o processo preferencial mediado pela proteína clatrina.

Neste trabalho foi ainda possível identificar alguns alvos intracelulares; por Microscopia Electrónica de Transmissão (TEM) observou-se que os compostos estudados provocam alterações no complexo de Golgi e nas mitocôndrias 
podendo estas alterações estar relacionadas com o mecanismo de morte celular. Os estudos por espectrometria de massa também permitiram identificar alguns alvos intracelulares para estes compostos, nomeadamente as proteínas ubiquitina e citocromo $c$ e o enzima superóxido dismutase.

\section{EFEITO TERAPÊUTICO IN VIVO}

De modo a obter informações sobre o desempenho in vivo destes complexos foram realizados estudos em modelos animais (ratinhos nude) inoculados com tumores dos cancros da próstata e mama. O estudo mais relevante relativo ao efeito terapêutico de um destes compostos foi avaliado num modelo animal com uma implantação ortotópica de células do cancro da mama triplo negativo. Estes tumores são caracterizados por serem altamente agressivos, terem um elevado risco de recorrência e um elevado potencial metastático (especialmente para os pulmões) resultando, na maioria dos casos, em morte precoce. Os resultados obtidos mostram que o complexo de ruténio estudado é bem tolerado pelos animais e não apresenta os fortes efeitos secundários observados para outros metalofármacos, como a cisplatina (usada também nestes estudos para comparação). Além disso, os nossos estudos mostraram claramente que este composto é um eficiente supressor do crescimento in vivo dos tumores induzidos pelas células de cancro da mama MDAMB231 e a autópsia dos animais não revelou a presença de quaisquer metástases nos pulmões ou outros órgãos. Mais ainda, para todos os órgãos analisados histologicamente (pulmões, rins, fígado e baço) não foram encontrados quaisquer sinais de lesão. É importante realçar que o tratamento contínuo com o complexo de ruténio, após a remoção do tumor, aumentou drasticamente o tempo de vida dos animais que não apresentaram recorrência do tumor ou metástases.

\section{ConclusÃo}

Os novos compostos desenvolvidos combinam uma elevada actividade citotóxica in vitro para seis linhas celulares tumorais humanas, com importante selectividade para as células cancerígenas e um bom grau de inocuidade para as células saudáveis. Esta característica é essencial para a diminuição da resistência ao tratamento por quimioterapia e diminuição dos efeitos secundários vulgarmente observados.

Dos estudos in vitro, relativos à compreensão do modo de acção destes potenciais fármacos, foi possível concluir que os complexos podem ser transportados na corrente sanguínea pela albumina e transferrina, que a sua entrada nas células é por processos de endocitose, e que o mecanismo de morte celular poderá estar relacionado com alterações causadas pelos complexos no aparelho de Golgi e na mitocôndria.

Os estudos in vivo realizados em tumores da mama de extrema malignidade mostraram que os compostos suprimem eficientemente o crescimento de tumores e metástases, sem quaisquer sinais de lesão dos principais órgãos.

Este trabalho pode ser considerado como um passo em frente na descoberta de potenciais fármacos para quimioterapia.

\section{Agradecimentos}

À minha orientadora, Prof. ${ }^{a}$ Maria Helena Garcia. À Prof. ${ }^{a}$ Paula Robalo (ISEL/CQE-IST), às Doutoras Fernanda Marques e Lurdes Gano ( $\mathrm{C}^{2} \mathrm{TN}$ ), ao Dr. Francisco Tortosa (FMUL), ao Doutor António Matos (CIIEG), à Prof. ${ }^{\text {a }}$ Fátima Gärtner e MSc. Nuno Mendes (IPATMUP) por me ajudarem na concretização deste trabalho. À FCT pela concessão da bolsa de doutoramento (SFRH/BD/45871/2008). Este trabalho encontra-se protegido por duas patentes (PCT/IB2013/052035 e PCT/IB2012/054914).

\section{REFERÊNCIAS}

[1] The World Health Organization website, http://www.who. int/ mediacentre/ factsheets/ fs297/en/index.html, 10 Julho 2013

[2] M. Malvezzi, P. Bertuccio, F. Levi, C. La Vecchia, E. Negri, Ann. Oncol. 24 (2013) 792-800

[3] C. Soussain, D. Ricard, J.R. Fike, J.J. Mazeron, D. Psimaras, J.Y. Delattre, Lancet 374 (2009) 1639-1651

[4] A. Urruticoechea, R. Alemany, J. Balart, A. Villanueva, F. Vinals, G. Capella, Curr. Pharm. Des. 16 (2010) 3-10

[5] M. Melchart, A. Habtemariam, S. Parsons, P.J. Sadler, J. Inorg. Biochem. 101 (2007) 1903-1912

[6] A. Bergamo, A. Masi, A.F. Peacock, A. Habtemariam, P.J. Sadler, G. Sava, J. Inorg. Biochem. 104 (2010) 79-86

[7] R.E. Morris, R.E. Aird, P.D. Murdoch, H.M. Chen, J. Cummings, N.D. Hughes, S. Parsons, G. Boyd, D.I. Jodrell, P.J. Sadler, J. Med. Chem. 44 (2001) 3616-3621

[8] C.A. Vock, W.H. Ang, C. Scolaro, A.D. Phillips, L. Lagopoulos, L. Juillerat-Jeanneret, G. Sava, R. Scopelliti, P.J. Dyson, J. Med. Chem. 50 (2007) 2166-2175

[9] E. Meggers, G.E. Atilla-Gokcumen, H. Bregman, J. Makismoska, S.P. Mulcahy, N. Pagano, D.S. Williams, Synlett (2007) 1177-1189

[10] P.J.A. Madeira, T.S. Morais, T.J.L. Silva, P. Florindo, M.H. Garcia, Rapid. Commun. Mass Spectrom. 26 (2012) 1675$-1686$

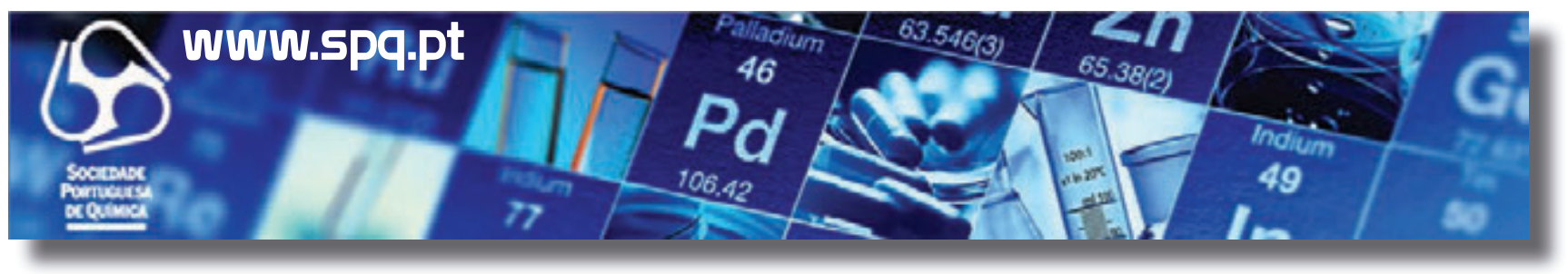

